\title{
A Green Ultrasound Synthesis, Characterization and Antibacterial Evaluation of 1,4-Disubstituted 1,2,3-Triazoles Tethering Bioactive Benzothiazole Nucleus
}

\author{
Nadjet Rezki ${ }^{1,2}$ \\ 1 Department of Chemistry, Faculty of Sciences, Taibah University, Al-Madinah Al-Munawarah 30002, \\ Saudi Arabia; nadjetrezki@yahoo.fr; Tel.: +966-540-953-573 \\ 2 Laboratoire de Chimie \& Electrochimie des Complexes Métalliques (LCECM), USTO-MB, \\ Department of Chemistry, Faculty of Sciences, University of Sciences, and Technology Mohamed Boudiaf, \\ B.p. 1505 El M'nouar, Oran 31000, Algeria
}

Academic Editor: Richard A. Bunce

Received: 11 March 2016; Accepted: 12 April 2016; Published: 18 April 2016

\begin{abstract}
The synthesis of $N$-(benzo[d]thiazol-2-yl)-2-(4-substituted-1H-1,2,3-triazol-1-yl)acetamides $\mathbf{5 a}-\mathbf{r}$ via the 1,3-dipolar cycloaddition reaction between 2-azido- $N$-(benzo[ $d]$ thiazol-2-yl)acetamide derivatives $3 \mathbf{a}-\mathbf{c}$ and different alkynes were performed in the presence and absence of ultrasound irradiation. The synthesis was carried out using $t-\mathrm{BuOH} / \mathrm{H}_{2} \mathrm{O}(1: 1, v / v)$ as reaction solvents and $\mathrm{CuSO}_{4} \cdot 5 \mathrm{H}_{2} \mathrm{O} /$ sodium ascorbate as the catalyst. The copper catalyst was implemented to provide the regioselective 1,4-disubstituted 1,2,3-triazoles 5a-r. Significant reductions in reaction times with comparably higher yields were observed when the reactions were carried out under ultrasound irradiation. The structures of the newly synthesized 1,2,3-triazoles were elucidated by IR, NMR, MS, and elemental analyses. They were also screened for their antimicrobial activity against three gram-positive (Streptococcus pneumonia, Bacillus subtilis, and Staphylococcus aureus), three gram-negative (Pseudomonas aeuroginosa, Escherichia coli, and Klebsiella pneumonia), and two fungal strains (Aspergillus fumigates and Candida albicans). Most of the tested compounds displayed promising antimicrobial activities at a Minimum Inhibition Concentration (MIC) of 4-16 $\mu \mathrm{g} / \mathrm{mL}$.
\end{abstract}

Keywords: 1,2,3-triazole; benzothiazole; 1,3-dipolar cycloaddition; ultrasound; antimicrobial activity

\section{Introduction}

Since the conception of "click chemistry" by Sharpless and co-workers, the synthesis of 1,2,3-triazoles is well-known and has been thoroughly studied [1]. In these reactions, the 1,3-dipolar cycloaddition between organoazides and terminal alkynes carried out under copper-catalyzed conditions have been reported to afford the regioisomeric 1,4-disubstituted 1,2,3-triazoles as the single regioismers [2].

The 1,2,3-triazole core has been recognized as one of the most potent azoles with broad chemotherapeutic properties including antifungal [3], anticancer [4], antitubercular [5], antimalarial [6], anti-inflammatory [7], and antiviral [8] activities, along with the application of the concept of "click-synthesis" for their efficient and quick synthesis.

Benzothiazole and its derivatives have been widely recognized as privileged scaffolds in drug design associated with a wide spectrum of medicinal applications [9-11]. These include antimicrobial [12-16], anticancer [17-20], anthelmintic [21], and antidiabetic [22] activities.

Owing to the interesting advantages of ultrasound in modern heterocyclic synthesis, this eco-friendly approach has been the focus of numerous investigations by several research groups 
for the design of novel potentially active heterocycles [23,24]. The ultrasound method can serve not only as a good alternative but also facilitate reaction in shorter times with higher yields $[25,26]$.

In order to evaluate the synergistic effect of these heterocyclic moieties in a single molecular framework, and in continuation of the author's effort to design structurally diverse bioactive polyheterocyclic systems [27-29], reported herein is an eco-friendly click synthesis of a library of new regioselective 1,4-disubstituted 1,2,3-triazoles linked to a bioactive benzothiazole moiety through an acetamide linkage under both ultrasound and conventional thermal heating based on literature data [30-34]. The newly synthesized compounds were also subjected to an in vitro antimicrobial screening against several clinical bacterial and fungal strains.

\section{Results and Discussion}

\subsection{Chemistry}

The desired 1,2,3-triazoles-based benzothiazoles were designed and synthesized starting from 2-aminobenzothiazole derivatives $\mathbf{1 a}-\mathbf{c}$ as outlined in Schemes 1 and 2.

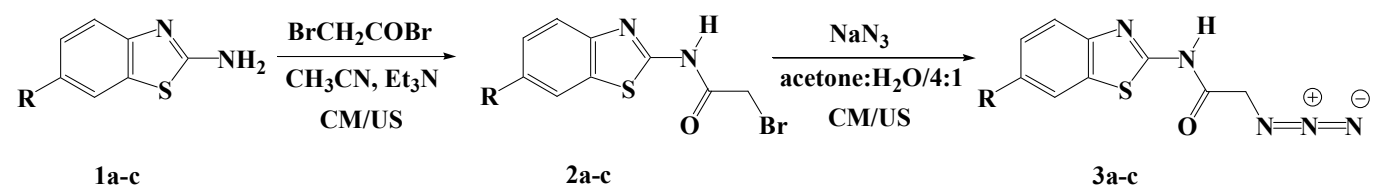

Scheme 1. Synthesis of azidobenzothiazoles 3a-c under ultrasound (US) and conventional methods (CM).
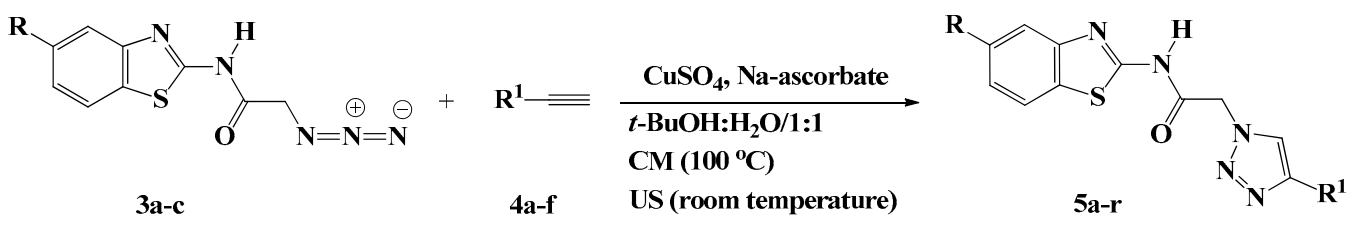

Scheme 2. Synthesis of 1,2,3-triazoles 5a-r based benzothiazole.

Compounds $\mathbf{2} \mathbf{a}-\mathbf{c}$ were employed for the synthesis of the starting azidobenzothiazoles $\mathbf{3 a}-\mathbf{c}$, needed for the 1,3-dipolar cycloaddition reaction. The synthesis of $N$-(benzo[d]thiazol-2-yl)-2bromoacetamides $\mathbf{2 a - c}$ was carried out based on a reported procedure [35] with slight modifications, as depicted in Scheme 1. Thus, compounds 2a-c were synthesized in $83 \%-88 \%$ yields via the acylation of un/substituted aminobenzothiazole 1a-c with bromoacetyl bromide, in the presence of triethylamine in acetonitrile at room temperature. The ultrasound irradiation was also used to construct the same products $\mathbf{2 a}-\mathbf{c}$ in shorter time $(1 \mathrm{~h})$ with higher product yields $(89 \%-92 \%)$ compared to the conventional methods (Table 1).

Table 1. Conventional versus ultrasound synthesis of compounds $\mathbf{2 a}-\mathbf{c}$ and $\mathbf{3 a}-\mathbf{c}$.

\begin{tabular}{cccccc}
\hline \multirow{2}{*}{ Compound No. } & \multirow{2}{*}{$\mathbf{R}$} & \multicolumn{2}{c}{ Classical Method CM } & \multicolumn{2}{c}{ Ultrasound Method US } \\
\cline { 3 - 6 } & & Time (h) & Yield (\%) & Time (h) & Yield (\%) \\
\hline $\mathbf{2 a}$ & $\mathrm{H}$ & 2 & 88 & 1 & 92 \\
$\mathbf{2 b}$ & $\mathrm{CH}_{3}$ & 2 & 86 & 1 & 91 \\
$\mathbf{2 c}$ & $\mathrm{CH}_{3} \mathrm{SO}_{2}$ & 3 & 83 & 1 & 89 \\
$\mathbf{3 a}$ & $\mathrm{H}$ & 24 & 92 & 2 & 96 \\
3b & $\mathrm{CH}_{3}$ & 24 & 91 & 2 & 96 \\
3c & $\mathrm{CH}_{3} \mathrm{SO}_{2}$ & 24 & 88 & 3 & 94 \\
\hline
\end{tabular}

The treatment of $\mathbf{2 a}-\mathbf{c}$ with sodium azide in a mixture of acetone:water $(4: 1, v / v)$ at room temperature for $24 \mathrm{~h}$ afforded the corresponding azidobenzothiazoles $3 \mathrm{a}-\mathrm{c}$ in good to excellent yields 
$(88 \%-92 \%)$, as shown in Scheme 1. It should be noted that compound 3a has been previously synthesized in $90 \%$ yield from the alkylation of $1 \mathbf{a}$ with the appropriate acid chloride in the presence of triethylamine in dimethylformamide (DMF), followed by nucleophilic substitution with sodium azide [36].

Alternatively, the ultrasound irradiation greatly accelerated the reaction rates under the same reaction conditions. Only 2-3 h were needed to furnish the azido compounds $3 a-c$ in high yields (Table 1).

Initial formation of the $\mathrm{N}$-(benzo[d]thiazol-2-yl)-2-bromoacetamides $\mathbf{2 a - c}$ was unambiguously confirmed by their spectroscopic data. Their IR spectra clearly showed the disappearance of the amino group and the appearance of strong absorptions near $1695-1700 \mathrm{~cm}^{-1}$ and $3277-3325 \mathrm{~cm}^{-1}$ characteristic of the carbonyl $(\mathrm{C}=\mathrm{O})$ and the amide $(\mathrm{NH})$ group, respectively. Moreover, the ${ }^{1} \mathrm{H}-\mathrm{NMR}$ spectra exhibited characteristic singlets at $\delta_{\mathrm{H}} 4.20-4.27 \mathrm{ppm}$ due to the methylene protons. The amide protons $(\mathrm{NH})$ were observed at $\delta_{\mathrm{H}} 6.42-6.48 \mathrm{ppm}$. The aromatic protons resonated at their expected chemical shift of $\delta_{\mathrm{H}} 7.22-8.64 \mathrm{ppm}$. In addition, the ${ }^{13} \mathrm{C}-\mathrm{NMR}$ spectra revealed characteristic signals between $\delta_{C} 28.5-41.7$ ppm belonging to the methylene groups. The spectra also revealed the appearance of new signals at $165.4-166.7 \mathrm{ppm}$ attributed to the carbonyl groups, which is another piece of evidence of the incorporation of the acetyl moiety in their structures.

The structures of the synthesized azido derivatives $3 \mathbf{a}-\mathbf{c}$ were also illustrated on the basis of their spectroscopic data. Their IR spectra exhibited an absorption band near $2105-2122 \mathrm{~cm}^{-1}$, confirming the presence of the azido group in their structures. Compounds $3 a-c$ exhibited practically similar ${ }^{1} \mathrm{H}-\mathrm{NMR}$ and ${ }^{13} \mathrm{C}-\mathrm{NMR}$ spectral patterns to those observed for their precursors $\mathbf{2 a - c}$ (See experimental section).

Huisgen copper(I)-catalyzed 1,3-dipolar cycloadditions between the azido benzothiazoles 3a-c and the appropriate terminal alkynes $4 \mathbf{a}-\mathbf{f}$ were performed under both conventional and ultrasound conditions to generate 5a-r, a new library of 1,4-disubstiuted 1,2,3-triazoles linked via an acetamide connecting unit to benzothiazoles (Scheme 2). The easy access to a variety of acetylenic compounds $(\mathbf{4} \mathbf{a}-\mathbf{f})$ allowed the incorporation of several functionalities into the triazole scaffold of $\mathbf{5 a}-\mathbf{r}$.

The reaction required heating at $100{ }^{\circ} \mathrm{C}$ for $6-10 \mathrm{~h}$, in the presence of a catalytic amount of copper sulfate $\left(\mathrm{CuSO}_{4}\right)$ and sodium ascorbate using $t-\mathrm{BuOH} / \mathrm{H}_{2} \mathrm{O}(1: 1, v / v)$ as solvents. Whereas only 3-6 h were needed to give the same products in comparable yields when the reactions were assisted by ultrasound irradiation at room temperature (Table 2). In the present study, $\mathrm{CuSO}_{4} /$ sodium ascorbate was selected as the catalyst system to yield the regioisomeric 1,4-disubstituted 1,2,3-triazoles as the sole regioisomer product. These results were in agreement with those previously reported for the successful green ultrasound-assisted synthesis of 1,4-disubstituted 1,2,3-triazoles, where the best results were obtained when using $t-\mathrm{BuOH} / \mathrm{H}_{2} \mathrm{O}$ as the medium and $\mathrm{CuSO}_{4}$ / sodium ascorbate as the catalyst [30-34].

Table 2. Conventional versus ultrasound synthesis of 1,2,3-triazoles 5a-r.

\begin{tabular}{|c|c|c|c|c|c|}
\hline \multirow{2}{*}{ Compound No } & \multirow{2}{*}{ Structure } & \multicolumn{2}{|c|}{ Classical Method CM } & \multicolumn{2}{|c|}{ Ultrasound Method US } \\
\hline & & Time (h) & Yield (\%) & Time (h) & Yield (\%) \\
\hline $5 a$ & & 6 & 90 & 3 & 96 \\
\hline $5 b$ & & 8 & 88 & 4 & 94 \\
\hline $5 c$ & & 7 & 89 & 4 & 96 \\
\hline
\end{tabular}


Table 2. Cont.

\begin{tabular}{|c|c|c|c|c|c|}
\hline \multirow{2}{*}{ Compound No } & \multirow{2}{*}{ Structure } & \multicolumn{2}{|c|}{ Classical Method CM } & \multicolumn{2}{|c|}{ Ultrasound Method US } \\
\hline & & Time (h) & Yield (\%) & Time (h) & Yield (\%) \\
\hline $5 d$ & & 9 & 85 & 5 & 90 \\
\hline $5 e$ & & 8 & 87 & 3 & 93 \\
\hline $5 f$ & & 8 & 87 & 3 & 92 \\
\hline $5 g$ & & 6 & 89 & 3 & 96 \\
\hline $5 h$ & & 8 & 88 & 4 & 93 \\
\hline $5 i$ & & 7 & 88 & 4 & 95 \\
\hline $5 \mathrm{j}$ & & 10 & 84 & 5 & 89 \\
\hline $5 \mathrm{k}$ & & 8 & 87 & 3 & 92 \\
\hline 51 & & 8 & 87 & 4 & 92 \\
\hline $5 \mathrm{~m}$ & & 8 & 81 & 4 & 92 \\
\hline $5 n$ & & 9 & 84 & 5 & 89 \\
\hline 50 & & 8 & 85 & 5 & 91 \\
\hline $5 p$ & & 10 & 81 & 6 & 87 \\
\hline
\end{tabular}


Table 2. Cont.

\begin{tabular}{cccccc}
\hline \multirow{2}{*}{ Compound No } & \multirow{2}{*}{ Structure } & \multicolumn{2}{c}{ Classical Method CM } & \multicolumn{2}{c}{ Ultrasound Method US } \\
\cline { 3 - 6 } & & Time (h) & Yield (\%) & Time (h) & Yield (\%) \\
\hline $5 \mathbf{9}$ & & 9 & 83 & 4 & 89 \\
\hline
\end{tabular}

All of the newly synthesized triazoles 5a-r were fully characterized using IR, NMR, and MS and elemental analysis. Their structures were in accordance with their spectroscopic properties. Their IR spectra showed the disappearance of the absorption bands attributed to the azido and the acetylenic groups of the corresponding starting materials, which confirmed their involvement in the cycloaddition reaction.

Moreover, the ${ }^{1} \mathrm{H}-\mathrm{NMR}$ spectra showed the absence of the acetylenic protons and the appearance of the diagnostic singlets at $\delta_{\mathrm{H}} 7.72-8.71$ and $\delta_{\mathrm{H}} 12.67-12.99 \mathrm{ppm}$ attributed to the triazolic proton and the amidic NH proton, respectively, supporting the proposed 1,2,3-triazole structures. In addition, their ${ }^{13} \mathrm{C}-\mathrm{NMR}$ spectra were consistent with the designed structures, the disappearance of the sp carbons provided additional evidence for the success of the cycloaddition reaction. In addition, the ${ }^{1} \mathrm{H}-\mathrm{NMR}$ spectra of compounds $\mathbf{5 a}-\mathbf{c}, \mathbf{5 g}-\mathbf{i}$, and $\mathbf{5 m}-\mathbf{o}$ revealed the presence of five and/or ten extra aromatic protons, depending on the nature of substitution on $\mathrm{N}-1$ of the triazole ring. The characteristic signals at $\delta_{\mathrm{H}} 4.50-6.80 \mathrm{ppm}$ were attributed to the 1,2,3-triazole derivatives carrying the hydroxy group.

In addition, all aliphatic protons and carbons resonated at the expected chemical shifts in the ${ }^{1} \mathrm{H}-\mathrm{NMR}$ spectra of triazoles $\mathbf{5} \mathbf{d}, \mathbf{5} \mathbf{j}$ and $\mathbf{5 p}$, the trimethylsilyl group appeared at $\delta_{\mathrm{H}} 0.28-0.34 \mathrm{ppm}$ corresponding to nine protons, and at $\delta_{C} 0.3-0.5 \mathrm{ppm}$ in the ${ }^{13} \mathrm{C}-\mathrm{NMR}$ spectra for the three methyl groups.

\subsection{Biology}

\section{Antimicrobial Activity}

The antibacterial and antifungal inhibition effects of the title compounds $\mathbf{2 a}-\mathbf{c}, \mathbf{3 a}-\mathbf{c}$ and $\mathbf{5 a}-\mathbf{r}$ against a panel of pathogenic bacterial and fungal strains were determined using the broth dilution method [37,38]. The Minimum Inhibition Concentration (MIC) screening results are given in Table 3, and Ciprofloxacin and Fluconazole were used as control drugs. The antimicrobial screening results revealed that the precursors $2 \mathbf{a}-\mathbf{c}$ used for further derivatization were basically inactive against all of the fungal strains, but exhibited modest antibacterial activity at MIC $16-31.25 \mu \mathrm{g} / \mathrm{mL}$.

The azidolysis of compounds $\mathbf{2 a}-\mathbf{c}$ to their corresponding azido benzothiazoles $3 \mathbf{a}-\mathbf{c}$ caused no enhancement of the antibacterial and antifungal activities. However, as expected, the 1,2,3-triazoles $\mathbf{5 a}-\mathbf{r}$ were more potent than the corresponding azidobenzothiazoles $3 \mathbf{a}-\mathbf{c}$. Among them, $\mathrm{N}-1$ hydroxylated alkyl substituted triazoles 5e, 5f, 5k, 51, 5q, and $5 \mathbf{r}$ exhibited the highest antibacterial activity at MIC $4-8 \mu \mathrm{g} / \mathrm{mL}$ and antifungal activity at MIC $4-16 \mu \mathrm{g} / \mathrm{mL}$.

Moreover, the presence of a sulfonyl group in the benzothiazole residue of compounds 4a-r was found to significantly increase the antimicrobial activity towards all of the tested pathogenic strains at MIC $4-16 \mu \mathrm{g} / \mathrm{mL}$.

Based on the preliminary structure-activity relationship analysis, it can be stated that the incorporation of a 1,2,3-triazole nucleus in the benzothiazole structure significantly improved the antimicrobial activities of the resulting biheterocyclic systems 5a-r, especially when the 1,2,3-triazole was substituted with hydroxylated alkyl side chain at position 1 . In addition, it must be noted that 
among all of the tested compounds, those with methylsulfonyl substitution in the benzothiazole ring $5 q$ and $5 r$ exhibited the highest inhibition against all of the tested microorganisms.

Table 3. Antimicrobial activity expressed as Minimum Inhibition Concentration (MIC, $\mu \mathrm{g} / \mathrm{mL}$ ).

\begin{tabular}{|c|c|c|c|c|c|c|c|c|}
\hline \multirow{2}{*}{$\begin{array}{c}\text { Compound } \\
\text { No }\end{array}$} & \multicolumn{3}{|c|}{ Gram-Positive Organisms } & \multicolumn{3}{|c|}{ Gram-Negative Organisms } & \multicolumn{2}{|c|}{ Fungi } \\
\hline & $S p$ & Bs & $S a$ & $P a$ & Ec & $K p$ & $A f$ & $\mathrm{Ca}$ \\
\hline $2 a$ & 31.25 & 31.25 & 16 & 31.25 & 16 & 31.25 & 125 & 62.5 \\
\hline $2 b$ & 31.25 & 31.25 & 16 & 16 & 16 & 31.25 & 125 & 125 \\
\hline $2 c$ & 16 & 31.25 & 16 & 16 & 16 & 16 & 125 & 62.5 \\
\hline $3 \mathbf{a}$ & 31.25 & 16 & 16 & 16 & 16 & 31.25 & 62.5 & 31.25 \\
\hline $3 b$ & 31.25 & 16 & 16 & 16 & 16 & 31.25 & 31.25 & 31.25 \\
\hline $3 c$ & 16 & 16 & 16 & 16 & 16 & 16 & 31.25 & 31.25 \\
\hline $5 a$ & 16 & 16 & 16 & 16 & 16 & 16 & 31.25 & 31.25 \\
\hline $5 b$ & 16 & 16 & 16 & 16 & 16 & 16 & 31.25 & 31.25 \\
\hline $5 c$ & 16 & 8 & 8 & 16 & 8 & 16 & 16 & 16 \\
\hline $5 d$ & 16 & 16 & 8 & 8 & 8 & 16 & 31.25 & 31.25 \\
\hline $5 e$ & 8 & 8 & 8 & 8 & 8 & 16 & 16 & 16 \\
\hline $5 f$ & 8 & 8 & 8 & 8 & 8 & 16 & 16 & 16 \\
\hline $5 \mathrm{~g}$ & 16 & 16 & 16 & 16 & 16 & 16 & 31.25 & 31.25 \\
\hline $5 \mathrm{~h}$ & 16 & 16 & 16 & 16 & 16 & 16 & 31.25 & 31.25 \\
\hline $5 i$ & 8 & 8 & 8 & 16 & 8 & 8 & 16 & 16 \\
\hline $5 g$ & 16 & 8 & 8 & 8 & 8 & 8 & 31.25 & 31.25 \\
\hline $5 \mathrm{k}$ & 8 & 8 & 8 & 8 & 8 & 8 & 16 & 16 \\
\hline 51 & 8 & 8 & 8 & 8 & 8 & 8 & 16 & 16 \\
\hline $5 \mathrm{~m}$ & 8 & 8 & 16 & 8 & 8 & 16 & 16 & 16 \\
\hline $5 n$ & 8 & 16 & 8 & 8 & 8 & 16 & 16 & 16 \\
\hline 50 & 8 & 8 & 4 & 4 & 8 & 8 & 8 & 8 \\
\hline $5 p$ & 8 & 8 & 8 & 16 & 8 & 8 & 16 & 8 \\
\hline $5 q$ & 4 & 4 & 8 & 4 & 4 & 8 & 4 & 4 \\
\hline $5 r$ & 4 & 4 & 8 & 4 & 4 & 8 & 4 & 4 \\
\hline Ciprofloxacin & $\leqslant 4$ & $\leqslant 1$ & $\leqslant 4$ & $\leqslant 4$ & $\leqslant 1$ & $\leqslant 1$ & - & - \\
\hline Fluconazole & - & - & - & - & - & - & $\leqslant 1$ & $\leqslant 1$ \\
\hline
\end{tabular}

Sp: Streptococcus pneumonia; Bs: Bacillus subtilis; Sa: Staphylococcus aureus; Pa: Pseudomonas aeuroginosa; Ec: Escherichia coli; Kp: Klebsiella pneumonia; Af: Aspergillus fumigates; Ca: Candida albicans.

\section{Experimental Section}

\subsection{General}

All melting points were measured on a variable heater (Stuart, UK) melt-temp apparatus and are uncorrected. Sonochemical reactions were performed in a Kunshan KQ-250B ultrasound cleaner (50 KHz, $240 \mathrm{~W}$, Kunshan, China). The NMR spectra were measured with an Avance Bruker spectrotometer (Fällanden, Switzerland) at $400 \mathrm{MHz}$ for the ${ }^{1} \mathrm{H}-\mathrm{NMR}$ analysis and at $100 \mathrm{MHz}$ for the ${ }^{1} \mathrm{H}-\mathrm{NMR}$ analysis, using Tetramethylsilane (TMS) $(0.00 \mathrm{ppm})$ as the internal standard and DMSO- $d_{6}$ as a solvent. The IR spectra were measured in a KBr matrix with a Perkin-Elmer 1430 series FTIR spectrometer (Boston, MA, USA). A Finnigan MAT 95XL spectrometer (Darmstadt, Germany) was used for the determination of the EI mass spectra. Elemental analyses were performed using a GmbH-Vario EL III Element Analyzer (Munich, Germany).

\subsection{General Procedure for the Synthesis of 2-Bromo-N-(6-un/substitutedbenzo[d]thiazol-2-yl)acetamide 2a-c}

Method a: Bromoacetyl bromide $(1.2 \mathrm{mmol})$ was added dropwise to a mixture of 2-aminobenzothiazole derivatives $1 \mathrm{a}-\mathrm{c}(1 \mathrm{mmol})$ and triethylamine $(1.2 \mathrm{mmol})$ in acetonitrile $(15 \mathrm{~mL})$ at room temperature with stirring. The stirring was continued at room temperature for $2-3 \mathrm{~h}$. The $N$-(benzo[d]thiazol-2-yl)-2-bromoacetamide thus formed was filtered, washed with water, and recrystallized from ethanol. 
Method b: A mixture of 2-aminobenzothiazole derivatives 1a-c (1 mmol) in acetonitrile (15 mL), triethylamine $(1.2 \mathrm{mmol})$ and bromoacetyl bromide $(1.2 \mathrm{mmol})$ was sonicated for $1 \mathrm{~h}$ at room temperature under inert atmosphere in a laboratory ultrasonic cleaning bath. The reaction was treated as described above.

N-(Benzo[d]thiazol-2-yl)-2-bromoacetamide (2a). Colorless needles, m.p. $175-176{ }^{\circ}$ C; (Lit. m.p. 1730-174 $\left.{ }^{\circ} \mathrm{C}\right)$ [35]. IR $\left(v, \mathrm{~cm}^{-1}\right): 1580(\mathrm{C}=\mathrm{C}), 1625(\mathrm{C}=\mathrm{N}), 1695(\mathrm{C}=\mathrm{O}), 2948(\mathrm{C}-\mathrm{H}$ al), $3053(\mathrm{C}-\mathrm{H}$ ar), 3290 (N-H). ${ }^{1} \mathrm{H}-\mathrm{NMR}: \delta 4.26$ (s, 2H, $\left.\mathrm{CH}_{2}\right), 6.43$ (bs, 1H, NH), 7.30-7.36 (m, 1H, Ar-H), 7.43-7.49 $(\mathrm{m}, 1 \mathrm{H}, \mathrm{Ar}-\mathrm{H}), 7.75-7.80(\mathrm{~m}, 1 \mathrm{H}, \mathrm{Ar}-\mathrm{H}), 7.99-8.03$ (m, 1H, Ar-H). ${ }^{13} \mathrm{C}-\mathrm{NMR}: \delta 28.5\left(\mathrm{CH}_{2}\right), 120.6,121.7$, 123.7, 126.2, 131.4, 148.3, 157.5, 166.0 (Ar-C, C=N, C=O) ppm. EI MS (m/z): $269.81\left(\mathrm{M}^{+}\right)$. Anal. Calcd for $\mathrm{C}_{9} \mathrm{H}_{7} \mathrm{BrN}_{2} \mathrm{OS}$ : C 39.87; H 2.60; N 10.33. Found: C 39.72; H 2.68; N 10.40.

2-Bromo-N-(6-methylbenzo[d]thiazol-2-yl)acetamide (2b). Colorless needles, m.p. $157-158^{\circ} \mathrm{C} . \mathrm{IR}\left(v, \mathrm{~cm}^{-1}\right)$ : $1564(\mathrm{C}=\mathrm{C}), 1610(\mathrm{C}=\mathrm{N}), 1700(\mathrm{C}=\mathrm{O}), 2925$ (C-H al), $3084\left(\mathrm{C}-\mathrm{H}\right.$ ar), $3277(\mathrm{~N}-\mathrm{H}) .{ }^{1} \mathrm{H}-\mathrm{NMR}: \delta 2.44$ (s, 3H, $\left.\mathrm{CH}_{3}\right), 4.20\left(\mathrm{~s}, 2 \mathrm{H}, \mathrm{CH}_{2}\right), 6.48(\mathrm{~s}, 1 \mathrm{H}, \mathrm{NH}), 7.22(\mathrm{~d}, 1 \mathrm{H}, \mathrm{J}=8 \mathrm{~Hz}, \mathrm{Ar}-\mathrm{H}), 7.63(\mathrm{~d}, 1 \mathrm{H}, \mathrm{J}=8 \mathrm{~Hz}, \mathrm{Ar}-\mathrm{H}), 7.75$ (s, 1H, Ar-H). ${ }^{13} \mathrm{C}-\mathrm{NMR}: \delta 20.8\left(\mathrm{CH}_{3}\right), 31.6\left(\mathrm{CH}_{2}\right), 120.4,122.6,123.2,126.0,127.8,131.4,147.4,165.4$ (Ar-C, C=N, C=O) ppm. EI MS ( $m / z): 284.06\left(\mathrm{M}^{+}\right)$. Anal. Calcd for $\mathrm{C}_{10} \mathrm{H}_{9} \mathrm{BrN}_{2} \mathrm{OS}: \mathrm{C} 42.12 ; \mathrm{H}, 3.18 ; \mathrm{N}$, 9.82. Found: C 42.12; H, 3.18; N, 9.82.

2-Bromo-N-(6-(methylsulfonyl)benzo[d] thiazol-2-yl)acetamide (2c). Colorless needles, m.p. $211-212^{\circ} \mathrm{C}$. IR $\left(v, \mathrm{~cm}^{-1}\right): 1572(\mathrm{C}=\mathrm{C}), 1628(\mathrm{C}=\mathrm{N}), 1698(\mathrm{C}=\mathrm{O}), 2970\left(\mathrm{C}-\mathrm{H}\right.$ al), 3019 (C-H ar), $3325(\mathrm{~N}-\mathrm{H}) .{ }^{1} \mathrm{H}-\mathrm{NMR}: \delta$ 3.20 (s, 3H, CH $\mathrm{CH}_{3}, 4.27$ (s, 2H, $\left.\mathrm{CH}_{2}\right), 6.42$ (bs, 1H, NH), $7.84(\mathrm{~d}, 1 \mathrm{H}, \mathrm{J}=8 \mathrm{~Hz}, \mathrm{Ar}-\mathrm{H}), 7.94(\mathrm{~d}, 1 \mathrm{H}, J=8 \mathrm{~Hz}$, Ar-H), 8.64 (s, 1H, Ar-H). ${ }^{13} \mathrm{C}-\mathrm{NMR}: \delta 32.5\left(\mathrm{CH}_{3}\right), 41.7\left(\mathrm{CH}_{2}\right), 122.5,123.8,126.0,128.1,129.9,144.6$, 152.8, 166.7 (Ar-C, C=N, C=O) ppm. EI MS (m/z): $347.79\left(\mathrm{M}^{+}\right)$. Anal. Calcd for $\mathrm{C}_{10} \mathrm{H}_{9} \mathrm{BrN}_{2} \mathrm{O}_{3} \mathrm{~S}_{2}: \mathrm{C}$ 34.39; H 2.60; N 8.02. Found: C 34.30; H 2.48; N 8.15.

\subsection{General Procedure for the Synthesis of Azido Benzothiazole Derivatives 3a-c}

Method a: A mixture of compounds $2 \mathbf{a}-\mathbf{c}(1 \mathrm{mmol})$ and sodium azide $(1.2 \mathrm{mmol})$ in a mixture of acetone:water $(4: 1)(10 \mathrm{~mL})$ was stirred for $24 \mathrm{~h}$ at room temperature. The excess of solvent was evaporated under vacuum. Products $\mathbf{3 a}-\mathbf{c}$ were collected by filtration, washed with water and recrystallized from ethanol.

Method b: A mixture of compounds $2 \mathbf{a}-\mathbf{c}(1 \mathrm{mmol})$ and sodium azide $(1.2 \mathrm{mmol})$ in a mixture of acetone:water (4:1) (10 mL) was sonicated for 2-3 h at room temperature under inert atmosphere in a laboratory ultrasonic cleaning bath. The reaction mixture was treated as described above.

2-Azido-N-(benzo[d]thiazol-2-yl)acetamide (3a). Colorless needles, m.p. 210-211 ${ }^{\circ}$ C; (Lit. m.p. 208-210 $\left.{ }^{\circ} \mathrm{C}[36]\right)$. IR $\left(v, \mathrm{~cm}^{-1}\right): 1572(\mathrm{C}=\mathrm{C}), 1617(\mathrm{C}=\mathrm{N}), 1698(\mathrm{C}=\mathrm{O}), 2105(-\mathrm{N}=\mathrm{N}=\mathrm{N}), 2923(\mathrm{C}-\mathrm{H}$ al), 3076 (C-H ar), 3315 (N-H). ${ }^{1} \mathrm{H}-\mathrm{NMR}: \delta 4.26$ (s, 2H, $\mathrm{CH}_{2}$ ), 7.36 (dd, 1H, J = 4, $\left.12 \mathrm{~Hz}, \mathrm{Ar}-\mathrm{H}\right), 7.47$ (dd, $1 \mathrm{H}, J=4,12 \mathrm{~Hz}, \mathrm{Ar}-\mathrm{H}), 7.78(\mathrm{~d}, 1 \mathrm{H}, J=8 \mathrm{~Hz}, \mathrm{Ar}-\mathrm{H}), 8.01$ (d, 1H, J = $8 \mathrm{~Hz}, \mathrm{Ar}-\mathrm{H}), 12.57$ (s, $1 \mathrm{H}, \mathrm{NH})$. ${ }^{13}$ C-NMR: $\delta 49.2\left(\mathrm{CH}_{2}\right), 119.1,120.2,122.2,124.6,129.9,147.2,155.9,166.1($ Ar-C, C=N, C=O) ppm. EI MS (m/z): $233.15\left(\mathrm{M}^{+}\right)$. Anal. Calcd for $\mathrm{C}_{9} \mathrm{H}_{7} \mathrm{~N}_{5} \mathrm{OS}$ : C 46.34; H 3.02; N 30.03. Found: C 46.51; H 3.14; N 30.20 .

2-Azido-N-(6-methylbenzo[d]thiazol-2-yl)acetamide (3b). Colorless needles, m.p. 229-230 ${ }^{\circ} \mathrm{C}$. IR $\left(v, \mathrm{~cm}^{-1}\right)$ : $1572(\mathrm{C}=\mathrm{C}), 1603(\mathrm{C}=\mathrm{N}), 1701(\mathrm{C}=\mathrm{O}), 2122(-\mathrm{N}=\mathrm{N}=\mathrm{N}), 2955(\mathrm{C}-\mathrm{H}$ al), 3027 (C-H ar), $3294(\mathrm{~N}-\mathrm{H})$. ${ }^{1} \mathrm{H}-\mathrm{NMR}: \delta 2.42\left(\mathrm{~s}, 3 \mathrm{H}, \mathrm{CH}_{3}\right), 4.24\left(\mathrm{~s}, 2 \mathrm{H}, \mathrm{CH}_{2}\right), 7.20(\mathrm{~d}, 1 \mathrm{H}, \mathrm{J}=8 \mathrm{~Hz}, \mathrm{Ar}-\mathrm{H}), 7.67(\mathrm{~d}, 1 \mathrm{H}, J=8 \mathrm{~Hz}$, Ar-H), 7.71 (s, 1H, Ar-H), 12.75 (s, 1H, NH). ${ }^{13} \mathrm{C}-\mathrm{NMR}: \delta 21.1\left(\mathrm{CH}_{3}\right), 33.2\left(\mathrm{CH}_{2}\right), 120.8,122.4,123.6$, 127.4, 127.9, 131.2, 148.8, 165.8 (Ar-C, C=N, C=O) ppm. EI MS (m/z): $246.97\left(\mathrm{M}^{+}\right)$. Anal. Calcd for $\mathrm{C}_{10} \mathrm{H}_{9} \mathrm{~N}_{5} \mathrm{OS}$ : C 48.57; H 3.67; N 28.32. Found: C 48.44; H 3.58; N 28.43.

2-Azido-N-(6-(methylsulfonyl)benzo[d] thiazol-2-yl)acetamide (3c). Colorless needles, m.p. $242-243^{\circ} \mathrm{C}$. IR $\left(v, \mathrm{~cm}^{-1}\right): 1585(\mathrm{C}=\mathrm{C}), 1616(\mathrm{C}=\mathrm{N}), 1713(\mathrm{C}=\mathrm{O}), 2105(-\mathrm{N}=\mathrm{N}=\mathrm{N}), 2939(\mathrm{C}-\mathrm{H}$ al), $3064(\mathrm{C}-\mathrm{H}$ ar $), 3307$ (N-H). ${ }^{1} \mathrm{H}-\mathrm{NMR}: \delta 3.16$ (s, 3H, $\left.\mathrm{CH}_{3}\right), 4.32$ (s, 2H, $\left.\mathrm{CH}_{2}\right), 7.86-7.96$ (m, 2H, Ar-H), 8.60 (s, 1H, Ar-H), 
$12.81(\mathrm{~s}, 1 \mathrm{H}, \mathrm{NH}) .{ }^{13} \mathrm{C}-\mathrm{NMR}: \delta 38.7\left(\mathrm{CH}_{3}\right), 50.9\left(\mathrm{CH}_{2}\right), 122.4,124.2,129.4,131.7,134.4,145.8,153.3,166.0$ $($ Ar-C, $\mathbf{C}=\mathrm{N}, \mathrm{C}=\mathrm{O})$ ppm. EI MS $(\mathrm{m} / z)$ : $311.18\left(\mathrm{M}^{+}\right)$. Anal. Calcd for $\mathrm{C}_{10} \mathrm{H}_{9} \mathrm{~N}_{5} \mathrm{O}_{3} \mathrm{~S}_{2}: \mathrm{C} 38.58 ; \mathrm{H} 2.91 ; \mathrm{N}$ 22.49. Found: C 38.69; H 2.98; N 22.62.

\subsection{General Procedures for the Click Synthesis of 1,2,3-Triazoles 5a-r}

Method a: To a stirring solution of equimolar amounts of azidobenzothiazole $3 \mathbf{a}-\mathrm{c}$ and terminal alkyne 4a-f dissolved in $t$ - $\mathrm{BuOH}$ and water (1:1), $\mathrm{CuSO}_{4}(0.01 \mathrm{eq})$ and Na-ascorbate (0.01 eq) were added. Stirring was continued for $6-10 \mathrm{~h}$ at $100{ }^{\circ} \mathrm{C}$, until the consumption of the starting material as indicated by thin layer chromatography (TLC). Saturated brine solution was added to the reaction mixture, then the crude was extracted with ethyl acetate $(3 \times 50 \mathrm{~mL})$ and dried over sodium sulfate. Removal of the solvent in vacuum gave the desired 1,2,3-triazole derivatives $5 \mathbf{a}-\mathbf{r}$ which were crystallized from ethanol.

Method b: A mixture of equimolar amounts of azidobenzothiazole $3 a-c$ and terminal alkyne 4a-f, $\mathrm{CuSO}_{4}(0.01 \mathrm{eq})$ and Na-ascorbate (0.01 eq) in $t$ - $\mathrm{BuOH}$ and water (1:1) was sonicated for 3-6 h at room temperature under inert atmosphere in a laboratory ultrasonic cleaning bath. The reaction mixture was treated as described above.

N-(Benzo[d] thiazol-2-yl)-2-(4-phenyl-1H-1,2,3-triazol-1-yl)acetamide (5a). Brown solid, m.p. 122-123 ${ }^{\circ} \mathrm{C}$. IR $\left(v, \mathrm{~cm}^{-1}\right)$ : $1566(\mathrm{C}=\mathrm{C}), 1607(\mathrm{C}=\mathrm{N}), 1695(\mathrm{C}=\mathrm{O}), 2965\left(\mathrm{C}-\mathrm{H}\right.$ al), 3038 (C-H ar), $3304(\mathrm{~N}-\mathrm{H}) .{ }^{1} \mathrm{H}-\mathrm{NMR}$ : $\delta 5.61\left(\mathrm{~s}, 2 \mathrm{H}, \mathrm{CH}_{2}\right), 7.31-7.38(\mathrm{~m}, 2 \mathrm{H}, \mathrm{Ar}-\mathrm{H}), 7.44-7.49(\mathrm{~m}, 3 \mathrm{H}, \mathrm{Ar}-\mathrm{H}), 7.81(\mathrm{~d}, 1 \mathrm{H}, J=8 \mathrm{~Hz}, \mathrm{Ar}-\mathrm{H}), 7.90$ $(\mathrm{d}, 2 \mathrm{H}, J=8 \mathrm{~Hz}, \mathrm{Ar}-\mathrm{H}), 8.01(\mathrm{~d}, 1 \mathrm{H}, J=8 \mathrm{~Hz}, \mathrm{Ar}-\mathrm{H}), 8.65$ (s, 1H, CH-1,2,3-triazole), 12.99 (s, 1H, NH). ${ }^{13}$ C-NMR: $\delta 51.7\left(\mathrm{CH}_{2}\right), 120.6,121.8,123.1,123.8,125.1,126.2,127.9,128.9,130.5,131.5,146.3,157.8$, 165.8 (Ar-C, C=N, C=O) ppm. EI MS (m/z): $335.18\left(\mathrm{M}^{+}\right)$. Anal. Calcd for $\mathrm{C}_{17} \mathrm{H}_{13} \mathrm{~N}_{5} \mathrm{OS}$ : C 60.88; H 3.91; N 20.88. Found: C 60.69; H 3.86; N 20.76.

$\mathrm{N}$-(Benzo[d]thiazol-2-yl)-2-(4-(hydroxydiphenylmethyl)-1H-1,2,3-triazol-1-yl)-acetamide (5b). Brown solid, m.p. $146-147^{\circ} \mathrm{C}$. IR $\left(v, \mathrm{~cm}^{-1}\right)$ : $1583(\mathrm{C}=\mathrm{C}), 1615(\mathrm{C}=\mathrm{N}), 1706(\mathrm{C}=\mathrm{O}), 2942(\mathrm{C}-\mathrm{H}$ al), $3079(\mathrm{C}-\mathrm{H}$ ar), 3314-3389 (N-H, O-H). ${ }^{1} \mathrm{H}-\mathrm{NMR}: \delta 5.53$ (s, 2H, CH$\left.{ }_{2}\right), 6.59$ (s, 1H, OH), 7.00-7.38 (m, 12H, Ar-H), 7.79-8.00 (m, 3H, CH-1,2,3-triazole, Ar-H), 12.82 (s, 1H, NH). ${ }^{13} \mathrm{C}-\mathrm{NMR}: \delta 49.4\left(\mathrm{CH}_{2}\right), 64.7(\mathrm{C}-\mathrm{OH})$, 119.3, 120.6, 121.2, 121.6, 122.7, 123.0, 123.9, 124.4, 125.3, 126.4, 128.2, 128.4, 131.7, 133.2, 147.1, 156.5, $166.3($ Ar-C, $\mathrm{C}=\mathrm{N}, \mathrm{C}=\mathrm{O})$ ppm. EI MS $(\mathrm{m} / z)$ : $441.02\left(\mathrm{M}^{+}\right)$. Anal. Calcd for $\mathrm{C}_{24} \mathrm{H}_{19} \mathrm{~N}_{5} \mathrm{O}_{2} \mathrm{~S}: \mathrm{C} 65.29 ; \mathrm{H}$ 4.34; N 15.86. Found: C 65.11; H 4.22; N 15.79.

N-(Benzo[d]thiazol-2-yl)-2-(4-(hydroxy(phenyl)methyl)-1H-1,2,3-triazol-1-yl)-acetamide (5c). Brown solid, m.p. $180-181^{\circ} \mathrm{C}$. IR (v, cm $\left.{ }^{-1}\right)$ : $1573(\mathrm{C}=\mathrm{C}), 1602(\mathrm{C}=\mathrm{N}), 1711(\mathrm{C}=\mathrm{O}), 2924(\mathrm{C}-\mathrm{H}$ al), $3082(\mathrm{C}-\mathrm{H}$ ar), 3287-3355 (N-H, O-H). ${ }^{1} \mathrm{H}-\mathrm{NMR}: \delta 5.42\left(\mathrm{~s}, 2 \mathrm{H}, \mathrm{CH}_{2}\right), 5.81(\mathrm{~d}, 1 \mathrm{H}, J=8 \mathrm{~Hz}, \mathrm{CH}), 5.97(\mathrm{~d}, 1 \mathrm{H}, J=8 \mathrm{~Hz}$, OH), 7.19-7.36 (m, 7H, Ar-H), 7.72-7.92 (m, 3H, CH-1,2,3-triazole, Ar-H), 12.75 (s, 1H, NH). ${ }^{13} \mathrm{C}-\mathrm{NMR}$ : $\delta 49.4\left(\mathrm{CH}_{2}\right), 64.7(\mathrm{C}-\mathrm{OH}), 119.6,120.8,121.1,122.8,123.2,124.7,125.9,126.7,128.8,131.5,132.0,146.6$, 156.8, 166.5 (Ar-C, C=N, C=O) ppm. EI MS ( $m / z)$ : $365.22\left(\mathrm{M}^{+}\right)$. Anal. Calcd for $\mathrm{C}_{18} \mathrm{H}_{15} \mathrm{~N}_{5} \mathrm{O}_{2} \mathrm{~S}: \mathrm{C} 59.16$; H 4.14; N 19.17. Found:C 59.45; H 4.26; N 19.43.

N-(Benzo[d]thiazol-2-yl)-2-(4-(trimethylsilyl)-1H-1,2,3-triazol-1-yl)acetamide (5d). Brown solid, m.p. 108-109 ${ }^{\circ} \mathrm{C}$. IR $\left(v, \mathrm{~cm}^{-1}\right): 1580(\mathrm{C}=\mathrm{C}), 1602(\mathrm{C}=\mathrm{N}), 1701(\mathrm{C}=\mathrm{O}), 2965(\mathrm{C}-\mathrm{H}$ al), 3051 (C-H ar), 3313 (N-H). ${ }^{1} \mathrm{H}-\mathrm{NMR}: \delta 0.29\left(\mathrm{~s}, 9 \mathrm{H}, 3 \times \mathrm{CH}_{3}\right), 5.56\left(\mathrm{~s}, 2 \mathrm{H}, \mathrm{CH}_{2}\right), 7.29-7.33(\mathrm{~m}, 1 \mathrm{H}, \mathrm{Ar}-\mathrm{H}), 7.40-7.46(\mathrm{~m}, 1 \mathrm{H}$, Ar-H), $7.81(\mathrm{~d}, 1 \mathrm{H}, J=8 \mathrm{~Hz}, \mathrm{Ar}-\mathrm{H}), 7.99(\mathrm{~d}, 1 \mathrm{H}, J=8 \mathrm{~Hz}, \mathrm{Ar}-\mathrm{H}), 8.20$ (s, 1H, CH-1,2,3-triazole), 12.89 (s, $1 \mathrm{H}, \mathrm{NH}) .{ }^{13} \mathrm{C}-\mathrm{NMR}: \delta 0.5\left(\mathrm{CH}_{3}\right), 50.9\left(\mathrm{CH}_{2}\right), 120.3,121.6,122.8,123.9,128.4,129.8,147.5,155.3,166.7$ $($ Ar-C, $\mathbf{C}=\mathrm{N}, \mathbf{C}=\mathrm{O})$ ppm. EI MS $(\mathrm{m} / z)$ : $331.24\left(\mathrm{M}^{+}\right)$. Anal. Calcd for $\mathrm{C}_{14} \mathrm{H}_{17} \mathrm{~N}_{5} \mathrm{OSSi}$ : C 50.73; $\mathrm{H}$ 5.17; $\mathrm{N}$ 21.13. Found: C 50.41; H 5.05; N 21.33.

N-(Benzo[d] thiazol-2-yl)-2-(4-(2-hydroxyethyl)-1H-1,2,3-triazol-1-yl)acetamide (5e). Brown solid, m.p. 228-229 ${ }^{\circ} \mathrm{C}$. IR $\left(v, \mathrm{~cm}^{-1}\right)$ : $1574(\mathrm{C}=\mathrm{C}), 1615(\mathrm{C}=\mathrm{N}), 1706(\mathrm{C}=\mathrm{O}), 2938(\mathrm{C}-\mathrm{H}$ al), $3060(\mathrm{C}-\mathrm{H}$ ar), 3302-3389 (N-H, O-H). ${ }^{1} \mathrm{H}-\mathrm{NMR}: \delta 2.81\left(\mathrm{t}, 2 \mathrm{H}, \mathrm{J}=8 \mathrm{~Hz}, \mathrm{C}-\mathrm{CH}_{2}\right), 3.67\left(\mathrm{t}, 2 \mathrm{H}, \mathrm{J}=8 \mathrm{~Hz}, \mathrm{OCH}_{2}\right), 4.72(\mathrm{~s}, 1 \mathrm{H}, \mathrm{OH})$, $5.50\left(\mathrm{~s}, 2 \mathrm{H}, \mathrm{CH}_{2}\right), 7.32-7.46$ (m, 2H, Ar-H), 7.80-7.95 (m, 3H, CH-1,2,3-triazole, Ar-H), 12.86 (s, 1H, 
NH). ${ }^{13} \mathrm{C}-\mathrm{NMR}: \delta 29.0\left(\mathrm{C}-\mathrm{CH}_{2}\right), 50.4\left(\mathrm{CH}_{2}\right), 60.3\left(\mathrm{OCH}_{2}\right), 120.8,121.3,122.6,123.1,127.7,129.3,146.1$, 156.7, 165.4 (Ar-C, C=N, C=O) ppm. EI MS ( $m / z)$ : $303.15\left(\mathrm{M}^{+}\right)$. Anal. Calcd for $\mathrm{C}_{13} \mathrm{H}_{13} \mathrm{~N}_{5} \mathrm{O}_{2} \mathrm{~S}$ : C 51.47; H 4.32; N 23.09. Found: C 51.62; H 4.28; N 23.18.

N-(Benzo[d] thiazol-2-yl)-2-(4-(3-hydroxypropyl)-1H-1,2,3-triazol-1-yl)acetamide (5f). Brown solid, m.p. 240-241 ${ }^{\circ} \mathrm{C}$. IR $\left(v, \mathrm{~cm}^{-1}\right): 1566(\mathrm{C}=\mathrm{C}), 1611(\mathrm{C}=\mathrm{N}), 1697$ (C=O), 2918 (C-H al), 3045 (C-H ar), 3274-3364 (N-H, O-H). ${ }^{1} \mathrm{H}-\mathrm{NMR}: \delta 1.76-1.79\left(\mathrm{~m}, 2 \mathrm{H}, \mathrm{OCH}_{2} \mathrm{CH}_{2}\right), 2.69\left(\mathrm{t}, 2 \mathrm{H}, J=8 \mathrm{~Hz}, \mathrm{C}-\mathrm{CH}_{2}\right), 3.47\left(\mathrm{~b}, 2 \mathrm{H}, \mathrm{OCH}_{2}\right.$ overlapped with DMSO), $4.50(\mathrm{~s}, 1 \mathrm{H}, \mathrm{OH}), 5.48\left(\mathrm{~s}, 2 \mathrm{H}, \mathrm{CH}_{2}\right), 7.34(\mathrm{dd}, 1 \mathrm{H}, J=4,8 \mathrm{~Hz}, \mathrm{Ar}-\mathrm{H}), 7.47$ (dd, $1 \mathrm{H}, J=4,8 \mathrm{~Hz}, \mathrm{Ar}-\mathrm{H}), 7.79(\mathrm{~d}, 1 \mathrm{H}, J=8 \mathrm{~Hz}, \mathrm{Ar}-\mathrm{H}), 7.92(\mathrm{~s}, 1 \mathrm{H}, \mathrm{CH}-1,2,3$-triazole), 7.99 (d, $1 \mathrm{H}, J=4 \mathrm{~Hz}$, $\mathrm{Ar}-\mathrm{H}), 12.81(\mathrm{~s}, 1 \mathrm{H}, \mathrm{NH}) .{ }^{13} \mathrm{C}-\mathrm{NMR}: \delta 29.0\left(\mathrm{OCH}_{2} \mathrm{CH}_{2}\right), 32.1\left(\mathrm{C}-\mathrm{CH}_{2}\right), 51.4\left(\mathrm{CH}_{2}\right), 60.0\left(\mathrm{OCH}_{2}\right), 120.7$, 121.7, 123.7, 123.9, 126.3, 129.4, 146.2, 156.0, 165.7 (Ar-C, C=N, C=O) ppm. EI MS $(m / z): 317.21\left(\mathrm{M}^{+}\right)$. Anal. Calcd for $\mathrm{C}_{14} \mathrm{H}_{15} \mathrm{~N}_{5} \mathrm{O}_{2} \mathrm{~S}$ : C 52.98; H 4.76; N 22.07. Found: C 52.80; H 4.59; N 22.28.

N-(6-Methylbenzo[d]thiazol-2-yl)-2-(4-phenyl-1H-1,2,3-triazol-1-yl)acetamide (5g). Brown solid, m.p. 133-134 ${ }^{\circ} \mathrm{C}$. IR $\left(v, \mathrm{~cm}^{-1}\right)$ : $1560(\mathrm{C}=\mathrm{C}), 1616(\mathrm{C}=\mathrm{N}), 1703(\mathrm{C}=\mathrm{O}), 2946(\mathrm{C}-\mathrm{H}$ al), $3077(\mathrm{C}-\mathrm{H}$ ar), $3345(\mathrm{~N}-\mathrm{H})$. ${ }^{1} \mathrm{H}-\mathrm{NMR}: \delta 2.41\left(\mathrm{~s}, 3 \mathrm{H}, \mathrm{CH}_{3}\right), 5.59$ (s, 2H, CH $\left.\mathrm{CH}_{2}\right), 7.27-7.37$ (m, 2H, Ar-H), 7.45-7.49 (m, 2H, Ar-H), 7.68 $(\mathrm{d}, 1 \mathrm{H}, J=8 \mathrm{~Hz}, \mathrm{Ar}-\mathrm{H}), 7.78$ (s, 1H, Ar-H), 7.89 (d, 2H, J = 8 Hz, Ar-H), 8.62 (s, 1H, CH-1,2,3-triazole), $12.86(\mathrm{~s}, 1 \mathrm{H}, \mathrm{NH}) .{ }^{13} \mathrm{C}-\mathrm{NMR}: \delta 20.9\left(\mathrm{CH}_{3}\right), 39.9\left(\mathrm{CH}_{2}\right), 121.3,123.0,125.1,127.5,127.9,128.6,130.2,133.7$, 147.4, 154.1, 165.4 (Ar-C, C=N, C=O) ppm. EI MS $(m / z)$ : $349.22\left(\mathrm{M}^{+}\right)$. Anal. Calcd for $\mathrm{C}_{18} \mathrm{H}_{15} \mathrm{~N}_{5} \mathrm{OS}: \mathrm{C}$ 61.87; H 4.33; N 20.04. Found: C 61.80; H 4.27; N 19.98.

2-(4-(Hydroxydiphenylmethyl)-1H-1,2,3-triazol-1-yl)-N-(6-methylbenzo[d]thiazol-2-yl)acetamide (5h). Brown solid, m.p. 148-149 ${ }^{\circ} \mathrm{C}$. IR (v, cm $\left.{ }^{-1}\right)$ : $1562(\mathrm{C}=\mathrm{C}), 1604(\mathrm{C}=\mathrm{N}), 1701(\mathrm{C}=\mathrm{O}), 2929(\mathrm{C}-\mathrm{H}$ al), $3036(\mathrm{C}-\mathrm{H}$ ar), 3287-3374 (N-H, O-H). ${ }^{1} \mathrm{H}-\mathrm{NMR}: \delta 2.41\left(\mathrm{~s}, 3 \mathrm{H}, \mathrm{CH}_{3}\right), 5.52\left(\mathrm{~s}, 2 \mathrm{H}, \mathrm{CH}_{2}\right), 6.60(\mathrm{~s}, 1 \mathrm{H}, \mathrm{OH}), 7.22-7.39$ (m, 12H, Ar-H), 7.77 (s, 1H, Ar-H), $7.92\left(\mathrm{~s}, 1 \mathrm{H}, \mathrm{CH}-1,2,3\right.$-triazole), $12.75(\mathrm{~s}, 1 \mathrm{H}, \mathrm{NH}) .{ }^{13} \mathrm{C}-\mathrm{NMR}: \delta 20.9$ $\left(\mathrm{CH}_{3}\right), 49.0\left(\mathrm{CH}_{2}\right), 75.6(\mathrm{C}-\mathrm{OH}), 121.3,125.0,125.5,126.7,126.9,127.5,127.9,128.5,129.5,133.3,147.0$, 153.7, 164.7 (Ar-C, C=N, C=O) ppm. EI MS ( $m / z)$ : $455.29\left(\mathrm{M}^{+}\right)$. Anal. Calcd for $\mathrm{C}_{25} \mathrm{H}_{21} \mathrm{~N}_{5} \mathrm{O}_{2} \mathrm{~S}: \mathrm{C} 65.92$; H 4.65; N 15.37. Found: C 65.78; H 4.53; N 15.26.

2-(4-(Hydroxy(phenyl)methyl)-1H-1,2,3-triazol-1-yl)-N-(6-methylbenzo[d] thiazol-2-yl)acetamide (5i). Brown solid, m.p. $162-163^{\circ} \mathrm{C}$. IR $\left(v, \mathrm{~cm}^{-1}\right)$ : $1570(\mathrm{C}=\mathrm{C}), 1607(\mathrm{C}=\mathrm{N}), 1701(\mathrm{C}=\mathrm{O}), 2924(\mathrm{C}-\mathrm{H}$ al), 3032 (C-H ar), 3275-3345 (N-H, O-H). ${ }^{1} \mathrm{H}-\mathrm{NMR}: \delta 2.45\left(\mathrm{~s}, 3 \mathrm{H}, \mathrm{CH}_{3}\right), 5.52\left(\mathrm{~s}, 2 \mathrm{H}, \mathrm{CH}_{2}\right), 5.91(\mathrm{~s}, 1 \mathrm{H}, J=4 \mathrm{~Hz}, \mathrm{CH}), 6.08$ $(\mathrm{s}, 1 \mathrm{H}, J=8 \mathrm{~Hz}, \mathrm{OH}), 7.30-7.50(\mathrm{~m}, 6 \mathrm{H}, \mathrm{Ar}-\mathrm{H}), 7.72(\mathrm{~d}, 1 \mathrm{H}, J=8 \mathrm{~Hz}, \mathrm{Ar}-\mathrm{H}), 7.81(\mathrm{~s}, 1 \mathrm{H}, \mathrm{Ar}-\mathrm{H}), 7.98(\mathrm{~s}$, 1H, CH-1,2,3-triazole), 12.75 (s, 1H, NH). ${ }^{13} \mathrm{C}-\mathrm{NMR}: \delta 20.0\left(\mathrm{CH}_{3}\right), 50,6\left(\mathrm{CH}_{2}\right), 67.0(\mathrm{C}-\mathrm{OH}), 119.3,120.4$, $122.8,125.4,126.1,126.6,127.1,127.2,130.6,132.4,143.1,150.5,164.8$ (Ar-C, C=N, C=O) ppm. EI MS $(m / z)$ : $379.00\left(\mathrm{M}^{+}\right)$. Anal. Calcd for $\mathrm{C}_{19} \mathrm{H}_{17} \mathrm{~N}_{5} \mathrm{O}_{2} \mathrm{~S}$ : C 60.14; H 4.52; N 18.46. Found: C 60.26; H 4.65; N 18.61 .

N-(6-Methylbenzo[d]thiazol-2-yl)-2-(4-(trimethylsilyl)-1H-1,2,3-triazol-1-yl)-acetamide (5j). Brown solid, m.p. 111-112 ${ }^{\circ} \mathrm{C}$. IR $\left(v, \mathrm{~cm}^{-1}\right)$ : 1583 (C=C), $1614(\mathrm{C}=\mathrm{N}), 1694$ (C=O), 2940 (C-H al), 3083 (C-H ar), 3316 (N-H). ${ }^{1} \mathrm{H}-\mathrm{NMR}: \delta 0.34\left(\mathrm{~s}, 9 \mathrm{H}, 3 \times \mathrm{CH}_{3}\right), 2.40\left(\mathrm{~s}, 3 \mathrm{H}, \mathrm{CH}_{3}\right), 5.50\left(\mathrm{~s}, 2 \mathrm{H}, \mathrm{CH}_{2}\right), 7.26(\mathrm{~d}, 1 \mathrm{H}, J=8 \mathrm{~Hz}$, Ar-H), 7.63 (d, 1H, J = $8 \mathrm{~Hz}, \mathrm{Ar}-\mathrm{H}), 7.70$ (s, 1H, Ar-H), 7.99 (s, 1H, CH-1,2,3-triazole), $12.84(\mathrm{~s}, 1 \mathrm{H}, \mathrm{NH})$. ${ }^{13} \mathrm{C}-\mathrm{NMR}: \delta 0.4\left(\mathrm{Si}-\mathrm{CH}_{3}\right), 20.4\left(\mathrm{CH}_{3}\right), 49.8\left(\mathrm{CH}_{2}\right), 120.3,121.7,123.6,126.9,130.3,132.0,146.7,154.0$, 165.9 (Ar-C, $\mathrm{C}=\mathrm{N}, \mathrm{C}=\mathrm{O}$ ) ppm. EI MS $(\mathrm{m} / z)$ ) $345.26\left(\mathrm{M}^{+}\right)$. Anal. Calcd for $\mathrm{C}_{15} \mathrm{H}_{19} \mathrm{~N}_{5} \mathrm{OSSi}$ : C 52.15; H 5.54; N 20.27. Found: C 52.43; H 5.43; N 20.39.

2-(4-(2-Hydroxyethyl)-1H-1,2,3-triazol-1-yl)-N-(6-methylbenzo[d]thiazol-2-yl)-acetamide (5k). Brown solid, m.p. $239-240{ }^{\circ} \mathrm{C}$. IR $\left(v, \mathrm{~cm}^{-1}\right)$ : $1592(\mathrm{C}=\mathrm{C}), 1603(\mathrm{C}=\mathrm{N}), 1711(\mathrm{C}=\mathrm{O}), 2965(\mathrm{C}-\mathrm{H}$ al), $3019(\mathrm{C}-\mathrm{H}$ ar), 3280-3375 (N-H, O-H). ${ }^{1} \mathrm{H}-\mathrm{NMR}: \delta 2.40\left(\mathrm{~s}, 3 \mathrm{H}, \mathrm{CH}_{3}\right), 2.82\left(\mathrm{t}, 2 \mathrm{H}, J=8 \mathrm{~Hz}, \mathrm{C}-\mathrm{CH}_{2}\right), 3.68\left(\mathrm{bt}, 2 \mathrm{H}, \mathrm{OCH}_{2}\right)$, $4.72(\mathrm{bs}, 1 \mathrm{H}, \mathrm{OH}), 5.47\left(\mathrm{~s}, 2 \mathrm{H}, \mathrm{CH}_{2}\right), 7.28(\mathrm{~d}, 1 \mathrm{H}, J=8 \mathrm{~Hz}, \mathrm{Ar}-\mathrm{H}), 7.67(\mathrm{~d}, 1 \mathrm{H}, J=8 \mathrm{~Hz}, \mathrm{Ar}-\mathrm{H}), 7.77$ (s, $1 \mathrm{H}, \mathrm{Ar}-\mathrm{H}), 7.94$ (s, 1H, CH-1,2,3-triazole), $12.77(\mathrm{~s}, 1 \mathrm{H}, \mathrm{NH}) .{ }^{13} \mathrm{C}-\mathrm{NMR}: \delta 20.9\left(\mathrm{CH}_{3}\right), 29.0\left(\mathrm{C}-\mathrm{CH}_{2}\right), 51.4$ $\left(\mathrm{CH}_{2}\right), 60.3\left(\mathrm{OCH}_{2}\right), 120.3,121.3,124.1,127.5,131.5,133.3,147.3,155.1,165.7(\mathrm{Ar}-\mathrm{C}, \mathrm{C}=\mathrm{N}, \mathrm{C}=\mathrm{O}) \mathrm{ppm}$. EI MS (m/z): $317.22\left(\mathrm{M}^{+}\right)$. Anal. Calcd for $\mathrm{C}_{14} \mathrm{H}_{15} \mathrm{~N}_{5} \mathrm{O}_{2} \mathrm{~S}$ : C 52.98; H 4.76; N 22.07. Found: $\mathrm{C} 52.77$; H 4.68; N 22.31. 
2-(4-(3-Hydroxypropyl)-1H-1,2,3-triazol-1-yl)-N-(6-ethylbenzo[d]thiazol-2-yl)-acetamide (51). Brown solid, m.p. $252-253^{\circ} \mathrm{C}$. IR $\left(v, \mathrm{~cm}^{-1}\right)$ : $1573(\mathrm{C}=\mathrm{C}), 1604(\mathrm{C}=\mathrm{N}), 1705(\mathrm{C}=\mathrm{O}), 2945(\mathrm{C}-\mathrm{H}$ al), $3067(\mathrm{C}-\mathrm{H}$ ar), 3280-3358 (N-H, O-H). ${ }^{1} \mathrm{H}-\mathrm{NMR}: \delta 1.73-1.80\left(\mathrm{~m}, 2 \mathrm{H}, \mathrm{OCH}_{2} \mathrm{CH}_{2}\right), 2.39\left(\mathrm{~s}, 3 \mathrm{H}, \mathrm{CH}_{3}\right), 2.68(\mathrm{t}, 2 \mathrm{H}, J=8 \mathrm{~Hz}$, $\left.\mathrm{C}-\mathrm{CH}_{2}\right), 3.45\left(\mathrm{~b}, 2 \mathrm{H}, \mathrm{OCH}_{2}\right.$ overlapped with DMSO), $4.53(\mathrm{~s}, 1 \mathrm{H}, \mathrm{OH}), 5.43\left(\mathrm{~s}, 2 \mathrm{H}, \mathrm{CH}_{2}\right), 7.24(\mathrm{~d}, 1 \mathrm{H}$, $J=8 \mathrm{~Hz}, \mathrm{Ar}-\mathrm{H}), 7.62(\mathrm{~d}, 1 \mathrm{H}, J=8 \mathrm{~Hz}, \mathrm{Ar}-\mathrm{H}), 7.72(\mathrm{~s}, 1 \mathrm{H}, \mathrm{Ar}-\mathrm{H}), 7.90$ (s, 1H, CH-1,2,3-triazole), 12.67 (bs, $1 \mathrm{H}, \mathrm{NH}) .{ }^{13} \mathrm{C}-\mathrm{NMR}: \delta 20.9\left(\mathrm{CH}_{3}\right), 21.6\left(\mathrm{OCH}_{2} \mathrm{CH}_{2}\right), 32.2\left(\mathrm{C}-\mathrm{CH}_{2}\right), 51.9\left(\mathrm{CH}_{2}\right), 60.0\left(\mathrm{OCH}_{2}\right), 117.3$, 120.0, 123.4, 126.4 127.2, 132.7, 146.6, 156.0, 165.7 (Ar-C, C=N, C=O) ppm. EI MS (m/z): $330.97\left(\mathrm{M}^{+}\right)$. Anal. Calcd for $\mathrm{C}_{15} \mathrm{H}_{17} \mathrm{~N}_{5} \mathrm{O}_{2} \mathrm{~S}$ : C 54.36; H 5.17; $\mathrm{N}$ 21.13. Found: $\mathrm{C} 54.58 ; \mathrm{H}$ 5.06; N 21.28.

N-(6-(Methylsulfonyl)benzo[d]thiazol-2-yl)-2-(4-phenyl-1H-1,2,3-triazol-1-yl)-acetamide (5m). Brown solid, m.p. $142-143^{\circ} \mathrm{C}$. IR $\left(v, \mathrm{~cm}^{-1}\right)$ : $1571(\mathrm{C}=\mathrm{C}), 1610(\mathrm{C}=\mathrm{N}), 1699(\mathrm{C}=\mathrm{O}), 2957(\mathrm{C}-\mathrm{H}$ al), 3026 (C-H ar), $3314(\mathrm{~N}-\mathrm{H}) .{ }^{1} \mathrm{H}-\mathrm{NMR}: \delta 2.98\left(\mathrm{~s}, 3 \mathrm{H}, \mathrm{CH}_{3}\right), 5.49\left(\mathrm{~s}, 2 \mathrm{H}, \mathrm{CH}_{2}\right), 7.26-7.34(\mathrm{~m}, 3 \mathrm{H}, \mathrm{Ar}-\mathrm{H}), 7.42-7.55(\mathrm{~m}$, 2H, Ar-H), 7.98 (d, 1H, J = $8 \mathrm{~Hz}, \mathrm{Ar}-\mathrm{H}), 8.05(\mathrm{~d}, 1 \mathrm{H}, J=8 \mathrm{~Hz}, \mathrm{Ar}-\mathrm{H}), 8.17(\mathrm{~s}, 1 \mathrm{H}, \mathrm{Ar}-\mathrm{H}), 8.68$ (s, 1H, CH-1,2,3-triazole), 12.76 (s, $1 \mathrm{H}, \mathrm{NH}) .{ }^{13} \mathrm{C}-\mathrm{NMR}: \delta 43.1\left(\mathrm{CH}_{3}\right), 48.2\left(\mathrm{CH}_{2}\right), 120.7,122.9,123.0,124.5$, 125.7, 126.2, 128.8, 130.4, 130.6, 145.6, 150.7, 154.3, 164.1 (Ar-C, C=N, C=O) ppm. EI MS (m/z): 413.14 $\left(\mathrm{M}^{+}\right)$. Anal. Calcd for $\mathrm{C}_{18} \mathrm{H}_{15} \mathrm{~N}_{5} \mathrm{O}_{3} \mathrm{~S}_{2}$ : C 52.29; H 3.66; N 16.94. Found: C 52.21; H 3.55; N 16.85.

2-(4-(Hydroxydiphenylmethyl)-1H-1,2,3-triazol-1-yl)-N-(6-(methylsulfonyl)benzo[d]-thiazol-2-yl)acetamide (5n). Brown solid, m.p. 154-155 ${ }^{\circ} \mathrm{C}$. IR $\left(v, \mathrm{~cm}^{-1}\right)$ : $1578(\mathrm{C}=\mathrm{C}), 1601(\mathrm{C}=\mathrm{N}), 1712(\mathrm{C}=\mathrm{O}), 2944$ (C-H al), 3060 (C-H ar), 3265-3349 (N-H, O-H). ${ }^{1} \mathrm{H}-\mathrm{NMR}: \delta 3.21$ (s, 3H, CH 3$), 5.46\left(\mathrm{~s}, 2 \mathrm{H}, \mathrm{CH}_{2}\right), 6.49$ (s, 1H, OH), 7.26-7.31 (m, 10H, Ar-H), 8.07 (d, 1H, J = $8 \mathrm{~Hz}, \mathrm{Ar}-\mathrm{H}), 8.21$ (d, 1H, J = $8 \mathrm{~Hz}, \mathrm{Ar}-\mathrm{H}), 8.25$ (s, 1H, Ar-H), 8.87 (s, 1H, CH-1,2,3-triazole), 12.68 (s, 1H, NH). ${ }^{13} \mathrm{C}-\mathrm{NMR}: \delta 40.3\left(\mathrm{CH}_{3}\right), 49.7\left(\mathrm{CH}_{2}\right), 79.1(\mathrm{C}-\mathrm{OH})$, $119.7,120.2,121.7,124.3,125.8,128.0,129.7,130.7,144.6,149.8,151.1,155.3,163.4($ Ar-C, C=N, C=O) ppm. EI MS (m/z): $519.02\left(\mathrm{M}^{+}\right)$. Anal. Calcd for $\mathrm{C}_{25} \mathrm{H}_{21} \mathrm{~N}_{5} \mathrm{O}_{4} \mathrm{~S}_{2}$ : C 57.79; $\mathrm{H} 4.07 ; \mathrm{N}$ 13.48. Found: $\mathrm{C}$ 57.88; H 4.19; N 13.64 .

2-(4-(Hydroxy(phenyl)methyl)-1H-1,2,3-triazol-1-yl)-N-(6-(methylsulfonyl)benzo[d]-thiazol-2-yl)acetamide (5o). Brown solid, m.p. 169-170 ${ }^{\circ} \mathrm{C}$. IR $\left(v, \mathrm{~cm}^{-1}\right): 1566(\mathrm{C}=\mathrm{C}), 1602(\mathrm{C}=\mathrm{N}), 1698(\mathrm{C}=\mathrm{O}), 2963(\mathrm{C}-\mathrm{H}$ al), 3069 (C-H ar), 3287-3360 (N-H, O-H). ${ }^{1} \mathrm{H}-\mathrm{NMR}: \delta 3.19$ (s, 3H, CH $\left.\mathrm{CH}_{3}\right), 5.59$ (s, 2H, CH $\left.\mathbf{C H}_{2}\right), 5.79$ (d, 1H, $J=4 \mathrm{~Hz}, \mathrm{CH}), 6.23(\mathrm{~s}, 1 \mathrm{H}, J=8 \mathrm{~Hz}, \mathrm{OH}), 7.25-7.41(\mathrm{~m}, 5 \mathrm{H}, \mathrm{Ar}-\mathrm{H}), 7.99(\mathrm{~d}, 1 \mathrm{H}, J=8 \mathrm{~Hz}, \mathrm{Ar}-\mathrm{H}), 8.14(\mathrm{~d}$, $1 \mathrm{H}, J=8 \mathrm{~Hz}, \mathrm{Ar}-\mathrm{H}), 8.17$ (s, 1H, Ar-H), 8.68 (s, 1H, CH-1,2,3-triazole), 12.72 (s, 1H, NH). ${ }^{13} \mathrm{C}-\mathrm{NMR}: \delta$ $41.3\left(\mathrm{CH}_{3}\right), 50.2\left(\mathrm{CH}_{2}\right), 69.2(\mathrm{C}-\mathrm{OH}), 120.4,121.8,124.4,127.1,127.5,128.6,129.8,141.2,145.9,147.0$, 155.3, 163.7 (Ar-C, $\mathrm{C}=\mathrm{N}, \mathrm{C}=\mathrm{O})$ ppm. EI MS $(\mathrm{m} / \mathrm{z})$ : $443.19\left(\mathrm{M}^{+}\right)$. Anal. Calcd for $\mathrm{C}_{19} \mathrm{H}_{17} \mathrm{~N}_{5} \mathrm{O}_{4} \mathrm{~S}_{2}$ : C 51.45; H 3.86; N 15.79. Found: C 51.31; H 3.70; N 15.68.

N-(6-(Methylsulfonyl)benzo[d]thiazol-2-yl)-2-(4-(trimethylsilyl)-1H-1,2,3-triazol-1-yl)acetamide (5p). Brown solid, m.p. 111-102 ${ }^{\circ} \mathrm{C}$. IR (v, cm $\left.{ }^{-1}\right)$ : 1569 (C=C), 1600 (C=N), 1698 (C=O), 2910 (C-H al), 3024 (C-H ar), $3346(\mathrm{~N}-\mathrm{H}) .{ }^{1} \mathrm{H}-\mathrm{NMR}: \delta 0.28\left(\mathrm{~s}, 9 \mathrm{H}, 3 \times \mathrm{CH}_{3}\right), 3.23\left(\mathrm{~s}, 3 \mathrm{H}, \mathrm{CH}_{3}\right), 5.62\left(\mathrm{~s}, 2 \mathrm{H}, \mathrm{CH}_{2}\right), 7.97(\mathrm{~d}, 2 \mathrm{H}, J=8 \mathrm{~Hz}$, Ar-H), 8.21 (s, 1H, Ar-H), 8.67 (s, 1H, CH-1,2,3-triazole), 12.91 (s, 1H, NH). ${ }^{13} \mathrm{C}-\mathrm{NMR}: \delta 0.3\left(\mathrm{Si}_{-} \mathrm{CH}_{3}\right)$, $40.0\left(\mathrm{CH}_{3}\right), 45.0\left(\mathrm{CH}_{2}\right), 120.4,121.7,123.8,128.5,130.6,135.4,146.6,156.7,163.5(\mathrm{Ar}-\mathrm{C}, \mathrm{C}=\mathrm{N}, \mathrm{C}=\mathrm{O}) \mathrm{ppm}$. EI MS ( $m / z)$ : $409.13\left(\mathrm{M}^{+}\right)$. Anal. Calcd for $\mathrm{C}_{15} \mathrm{H}_{19} \mathrm{~N}_{5} \mathrm{O}_{3} \mathrm{~S}_{2} \mathrm{Si}$ : C 43.99; $\mathrm{H} 4.68 ; \mathrm{N}$ 17.10. Found: C 43.74; H 4.79; N 17.31.

2-(4-(2-Hydroxyethyl)-1H-1,2,3-triazol-1-yl)-N-(6-(methylsulfonyl)benzo[d]thiazol-2-yl)acetamide (5q). Brown solid, m.p. 248-249 ${ }^{\circ} \mathrm{C}$. IR $\left(v, \mathrm{~cm}^{-1}\right): 1588(\mathrm{C}=\mathrm{C}), 1607(\mathrm{C}=\mathrm{N}), 1701(\mathrm{C}=\mathrm{O}), 2959$ (C-H al), 3069 (C-H ar), 3268-3354 (N-H, O-H). ${ }^{1} \mathrm{H}-\mathrm{NMR}: \delta 2.87\left(\mathrm{t}, 2 \mathrm{H}, J=8 \mathrm{~Hz}, \mathrm{C}-\mathrm{CH}_{2}\right), 3.27\left(\mathrm{~s}, 3 \mathrm{H}, \mathrm{CH}_{3}\right), 3.61\left(\mathrm{bt}, 2 \mathrm{H}, \mathrm{OCH}_{2}\right)$, 4.91 (bs, $1 \mathrm{H}, \mathrm{OH}), 5.49$ (s, 2H, CH$\left.{ }_{2}\right), 7.88(\mathrm{~d}, 1 \mathrm{H}, J=8 \mathrm{~Hz}, \mathrm{Ar}-\mathrm{H}), 8.04(\mathrm{~d}, 1 \mathrm{H}, J=8 \mathrm{~Hz}, \mathrm{Ar}-\mathrm{H}), 8.19$ (s, $1 \mathrm{H}, \mathrm{Ar}-\mathrm{H}), 8.71\left(\mathrm{~s}, 1 \mathrm{H}, \mathrm{CH}-1,2,3\right.$-triazole), $12.81(\mathrm{~s}, 1 \mathrm{H}, \mathrm{NH}) .{ }^{13} \mathrm{C}-\mathrm{NMR}: \delta 29.6\left(\mathrm{C}-\mathrm{CH}_{2}\right), 40.7\left(\mathrm{CH}_{3}\right), 51.9$ $\left(\mathrm{CH}_{2}\right), 60.8\left(\mathrm{OCH}_{2}\right), 120.2,121.9,124.7,128.0,131.5,145.6,153.5,158.8,163.4(\mathrm{Ar}-\mathrm{C}, \mathrm{C}=\mathrm{N}, \mathrm{C}=\mathrm{O}) \mathrm{ppm}$. EI MS $(m / z)$ : $380.93\left(\mathrm{M}^{+}\right)$. Anal. Calcd for $\mathrm{C}_{14} \mathrm{H}_{15} \mathrm{~N}_{5} \mathrm{O}_{4} \mathrm{~S}_{2}$ : $\mathrm{C} 44.08 ; \mathrm{H} 3.96 ; \mathrm{N}$ 18.36. Found: $\mathrm{C} 44.27$; H 3.99; N 18.48 .

2-(4-(3-Hydroxypropyl)-1H-1,2,3-triazol-1-yl)-N-(6-(methylsulfonyl)benzo[d]thiazol-2-yl)acetamide (5r). Brown solid, m.p. $267-268^{\circ} \mathrm{C}$. IR $\left(v, \mathrm{~cm}^{-1}\right)$ : $1568(\mathrm{C}=\mathrm{C}), 1611(\mathrm{C}=\mathrm{N}), 1721(\mathrm{C}=\mathrm{O}), 2963(\mathrm{C}-\mathrm{H}$ al), 
3042 (C-H ar), 3299-3374 (N-H, O-H). ${ }^{1} \mathrm{H}-\mathrm{NMR}: \delta 1.69-1.76\left(\mathrm{~m}, 2 \mathrm{H}, \mathrm{OCH}_{2} \mathrm{CH}_{2}\right), 2.71(\mathrm{t}, 2 \mathrm{H}, \mathrm{J}=8 \mathrm{~Hz}$, $\left.\mathrm{C}-\mathrm{CH}_{2}\right), 3.32\left(\mathrm{~s}, 3 \mathrm{H}, \mathrm{CH}_{3}\right), 3.52\left(\mathrm{~b}, 2 \mathrm{H}, \mathrm{OCH}_{2}\right.$ overlapped with DMSO), 4.59 (s, 1H, OH), 5.51 (s, $\left.2 \mathrm{H}, \mathrm{CH}_{2}\right), 7.84(\mathrm{~d}, 1 \mathrm{H}, J=8 \mathrm{~Hz}, \mathrm{Ar}-\mathrm{H}), 8.02(\mathrm{~d}, 1 \mathrm{H}, J=8 \mathrm{~Hz}, \mathrm{Ar}-\mathrm{H}), 8.15(\mathrm{~s}, 1 \mathrm{H}, \mathrm{Ar}-\mathrm{H}), 8.70(\mathrm{~s}, 1 \mathrm{H}$, CH-1,2,3-triazole), 12.78 (bs, $1 \mathrm{H}, \mathrm{NH}) .{ }^{13} \mathrm{C}-\mathrm{NMR}$ : $\delta 23.4\left(\mathrm{OCH}_{2} \mathrm{CH}_{2}\right), 30.7\left(\mathrm{C}-\mathrm{CH}_{2}\right), 42.6\left(\mathrm{CH}_{3}\right), 49.8$ $\left(\mathrm{CH}_{2}\right), 61.8\left(\mathrm{OCH}_{2}\right), 119.8,121.2,124.4,127.0,131.2,145.1,148.7,154.0,157.2,164.0(\mathrm{Ar}-\mathrm{C}, \mathrm{C}=\mathrm{N}, \mathrm{C}=\mathrm{O})$ ppm. EI MS ( $m / z): 395.20\left(\mathrm{M}^{+}\right)$. Anal. Calcd for $\mathrm{C}_{15} \mathrm{H}_{17} \mathrm{~N}_{5} \mathrm{O}_{4} \mathrm{~S}_{2}$ : C 45.56; $\mathrm{H} 4.33 ; \mathrm{N} 17.71$. Found: $\mathrm{C}$ 45.47; H 4.42; N 17.92.

\subsection{Antimicrobial Activity}

The antimicrobial inhibition potency of the newly synthesized benzothiazoles was estimated in terms of minimum inhibition concentration (MIC) by using the Broth Microdilution method [37,38]. Each compound was tested against clinical bacterial and fungal strains; (Streptococcus pneumonia RCMB 010010, Bacillus subtilis RCMB 010067, Staphylococcus aureus RCMB 010025, Pseudomonas aeuroginosa RCMB 010043, Escherichia coli RCMB 010052, Klebsiella pneumonia RCMB 010058, Aspergillus fumigates RCMB 02568 and Candida albicans RCMB 05036), and were obtained from the RCMB culture collection (Regional Center for Mycology and Biotechnology). The isolated clinical strains were subcultured on Mueller-Hinton Broth for bacteria and Sabouraud Liquid Broth for fungi. The stock solution of all compounds were prepared by dissolving $10 \mathrm{mg}$ of the tested compound in dimethyl sulfoxide (DMSO, $1 \mathrm{~mL}$ ). Progressive dilutions with distilled water gave the final concentrations of $1,2,4,8,16,31.25$, $62.5,125,250$, and $500 \mathrm{mg} \cdot \mathrm{mL}^{-1}$. All the inoculated tubes were incubated at $37^{\circ} \mathrm{C}$ for $24 \mathrm{~h}$.

\section{Conclusions}

This study reports on the synthesis of novel bioactive antibacterial and antifungal agents based on a 1,2,3-triazole-benzothiazole combined system under both conventional and ultrasound conditions. The synthesis approach required $\mathrm{Cu}(\mathrm{I})$-catalyzed 1,3-dipolar cycloaddition coupling between the appropriate 2-azido- $N$-(benzo[d]thiazol-2-yl)acetamides with a variety of terminal alkynes, to afford regioselectively novel 1,2,3-triazoles tethering a benzothiazole moiety. Comparable to higher yields were obtained when the reactions were conducted under ultrasound irradiation with a significant reduction in the reaction times. The antimicrobial screening results revealed that the presence of the 1,2,3-triazole nucleus in the acetamido-benzothiazole scaffold resulted in increased antibacterial and antifungal activities.

Acknowledgments: The author is very thankful to Meriem Amina Rezki, Faculty of Biotechnology, Es-Senia University, Oran, Algeria, for her assistance in the interpretation of the biological screening results.

Conflicts of Interest: The author declares no conflict of interest.

\section{References}

1. Rostovtsev, V.V.; Green, L.G.; Fokin, V.V.; Sharpless, K.B. A stepwise Huisgen cycloaddition process: copper(I)-catalyzed regioselective "ligation" of azides and terminal alkynes. Angew. Chem. Int. Ed. 2002, 41, 2596-2599. [CrossRef]

2. Xie, F.; Sivakumar, K.; Zeng, Q.; Bruckman, M.A.; Hodges, B.; Wang, Q. A fluorogenic 'click' reaction of azidoanthracene derivatives. Tetrahedron 2008, 64, 2906-2914. [CrossRef]

3. Aher, N.G.; Pore, V.S.; Mishra, N.N.; Kumar, A.; Shukla, P.K.; Sharma, A.; Bhat, M.K. Synthesis and antifungal activity of 1,2,3-triazole containing fluconazole analogues. Bioorg. Med. Chem. Lett. 2009, 19, 759-763. [CrossRef] [PubMed]

4. Kamal, A.; Shankaraiah, N.; Devaiah, V.; Reddy, K.L.; Juvekar, A.; Sen, S.; Kurian, N.; Zingde, S. Synthesis of 1,2,3-triazole-linked pyrrolobenzodiazepine conjugates employing 'click' chemistry: DNA-binding affinity and anticancer activity. Bioorg. Med. Chem. Lett. 2008, 18, 1468-1473. [CrossRef] [PubMed]

5. Singh, B.K.; Yadav, A.K.; Kumar, B.; Gaikwad, A.N.; Sinha, S.K.; Chaturvedi, V.; Tripathi, R.P. Preparation and reactions of sugar azides with alkynes: Synthesis of sugar triazoles as antitubercular agents. Carbohydr. Res. 2008, 343, 1153-1162. [CrossRef] [PubMed] 
6. Guantai, E.M.; Ncokaji, K.; Egan, T.J.; Gut, J.; Rosenthal, P.J.; Smith, P.J.; Chibale, K. Design, Synthesis and in vitro antimalarial evaluation of triazole-linked chalcone and dienone hybrid compounds. Bioorg. Med. Chem. 2010, 18, 8243-8256. [CrossRef] [PubMed]

7. Shafi, S.; Alam, M.M.; Mulakayala, N.; Mulakayala, C.; Vanaja, G.; Kalle, A.M.; Pallu, R.; Alam, M.S. Synthesis of novel 2-mercapto benzothiazole and 1,2,3-triazole based bis-heterocycles: Their anti-inflammatory and anti-nociceptive activities. Eur. J. Med. Chem. 2012, 49, 324-333. [CrossRef] [PubMed]

8. Piotrowska, D.G.; Balzarini, J.; Glowacka, I.E. Design, synthesis, antiviral and cytostatic evaluation of novel isoxazolidine nucleotide analogues with a 1,2,3-triazole linker. Eur. J. Med. Chem. 2012, 47, 501-509. [CrossRef] [PubMed]

9. Quiroga, J.; Hernandez, P.; Insuasty, B.; Abonıa, R.; Cobo, J.; Sanchez, A.; Nogueras, M.; Low, J.N. Control of the reaction between 2-aminobenzothiazoles and Mannich bases. Synthesis of pyrido[2,1-b][1,3]benzothiazoles versus [1,3]benzothiazolo[2,3-b]quinazolines. J. Chem. Soc. Perkin Trans. I 2002, 1, 555-559. [CrossRef]

10. Kok, S.H.L.; Chui, C.H.; Lam, W.S.; Chen, J.; Lau, F.Y.; Wong, R.S.M.; Cheng, G.Y.M.; Lai, P.B.S.; Leung, R.W.T.; Tang, J.C.O.; et al. Synthesis and structure evaluation of a novel cantharimide and its cytotoxicity on SK-Hep-1 hepatoma cells. Bioorg. Med. Chem. Lett. 2007, 17, 1155-1159. [CrossRef] [PubMed]

11. Kok, S.H.L.; Chui, C.H.; Lam, W.S.; Chen, J.; Lau, F.Y.; Wong, R.S.M.; Cheng, G.Y.M.; Tang, W.K.; Teo, I.T.N.; Cheung, F.; et al. Apoptogenic activity of a synthetic cantharimide in leukaemia: Implication on its structural activity relationship. Int. J. Mol. Med. 2006, 18, 1217-1221. [CrossRef] [PubMed]

12. Gupta, S.; Ajmera, N.; Gautam, N.; Sharma, R.; Gauatam, D. Novel synthesis and biological activity study of pyrimido [2,1-b] benzothiazoles. Ind. J. Chem. 2009, 48B, 853-858.

13. Kumbhare, R.M.; Ingle, V.N. Synthesis of novel benzothiozole and benzisoxazole functionalized unsymmetrical alkanes and study of their antimicrobial activity. Ind. J. Chem. 2009, 48B, 996-1000. [CrossRef]

14. Murthi, Y.; Pathak, D. Synthesis and Antimicrobial screening of Substituted 2-Mercaptobenzothiazoles. J. Pharm. Res. 2008, 7, 153-155.

15. Rajeeva, B.; Srinivasulu, N.; Shantakumar, S. Synthesis and Antimicrobial activity of some new 2-substituted benzothiazole derivatives. J. Chem. 2009, 6, 775-779.

16. Maharan, M.; William, S.; Ramzy, F.; Sembel, A. Synthesis and in vitro Evaluation of new benzothiazolederivaties as schistosomicidal agents. Molecules 2007, 12, 622-633. [CrossRef]

17. Kini, S.; Swain, S.; Gandhi, A. Synthesis and Evaluation of novel Benzothiazole Derivates against Human Cervical Cancer cell lines. Ind. J. Pharm. Sci. 2007, 46-50. [CrossRef]

18. Stanton, H.L.K.; Gambari, R.; Chung, H.C.; Johny, C.O.T.; Filly, C.; Albert, S.C.C. Synthesis and anti-cancer activity of benzothiazole containing phthalimide on human carcinoma cell lines. Bioorg. Med. Chem. 2008, 16, 3626-3631.

19. Wang, M.; Gao, M.; Mock, B.; Miller, K.; Sledge, G.; Hutchins, G.; Zheng, Q. Synthesis of C-11 labelled fluorinated 2-arylbenzothiazoles as novel potential PET cancer imaging agent. Bioorg. Med. Chem. 2006, 14, 8599-8607. [CrossRef] [PubMed]

20. Gupta, S.; Moorthi, N.; Sanyal, U. Synthesis, cytotoxic evaluation, in silico pharmacokinetic and QSAR study of some benzothiazole derivatives. Ind. J. Pharmacy Pharm. Sci. 2010, 2, 57-62.

21. Sreenivasa, M.; Jaychand, E.; Shivakumar, B.; Jayrajkumar, K.; Vijaykumar, J. Synthesis of bioactive molecule flurobenzothiazole comprising potent heterocylic moieties for anthelmintic activity. Arch. Pharm. Sci. Res. 2009, 1, 150-157.

22. Pattan, S.; Suresh, C.; Pujar, V.; Reddy, V.; Rasal, V.; Koti, B. Synthesis and antidiabetic activity of 2-amino[5"(4-sulphonylbenzylidine)-2,4-thiazolidinenone]-7-chloro-6-flurobenzothiazole. Ind. J. Chem. 2005, 44B, 2404-2408.

23. Cella, R.; Stefani, H.L.A. Ultrasound in heterocycles chemistry. Tetrahedron 2009, 65, 2619-2641. [CrossRef]

24. Alissa, S.A. Ultrasound Synthesis of Five-Membered Heterocycles. Chem. Sci. Rev. Lett. 2014, 3, 1219-1236.

25. Zbancioc, G.; Mangalagiu, I.I.; Moldoveanu, C. Ultrasound assisted synthesis of imidazolium salts: an efficient way to ionic liquids. Ultrason. Sonochem. 2015, 23, 376-384. [CrossRef] [PubMed]

26. Ameta, G.; Kumar Pathak, A.; Ameta, C.; Ameta, R.; Punjabi, P.B. Sonochemical synthesis and characterization of imidazolium based ionic liquids: A green pathway. J. Mol. Liq. 2015, 211, 934-937. [CrossRef] 
27. Aouad, M.R.; Rezki, N.; Kasmi, M.; Aouad, L.; Rezki, M.A. Synthesis, characterization and evaluation of antimicrobial activity of some novel 1,2,4-triazoles and 1,3,4-thiadiazoles bearing imidazole nuclues. Heterocycles 2012, 85, 1141-1154. [CrossRef]

28. Aouad, M.R.; Messali, M.; Rezki, N.; Ali, A.A.; Lesimple, A. Synthesis and characterization of some novel 1,2,4-triazoles, 1,3,4-thiadiazoles and Schiff bases incorporating imidazole moiety as potential antimicrobial agents. Acta Pharm. 2015, 65, 117-132. [CrossRef] [PubMed]

29. Rezki, N.; Al-Yahyawi, A.M.; Bardaweel, S.K.; Al-Blewi, F.F.; Aouad, M.R. Synthesis of Novel 2,5-Disubstituted-1,3,4-thiadiazoles Clubbed 1,2,4-Triazole, 1,3,4-Thiadiazole, 1,3,4-oxadiazole and/or Schiff Base as Potential Antimicrobial and Antiproliferative Agents. Molecules 2015, 20, 16048-16067. [CrossRef] [PubMed]

30. Cravotto, G.; Fokin, V.V.; Garella, D.; Binello, A.; Boffa, L.; Barge, A. Ultrasound-Promoted Copper-Catalyzed Azide-Alkyne Cycloaddition. J. Comb. Chem. 2010, 12, 13-15. [CrossRef] [PubMed]

31. Cintas, P.; Barge, A.; Tagliapietra, S.; Boffa, L.; Cravotto, G. Alkyne-azide click reaction catalyzed by metallic copper under ultrasound. Nat. Protoc. 2010, 5, 607-616. [CrossRef] [PubMed]

32. Jiang, Y.; Chen, X.; Qu, L.; Wang, J.; Yuan, J.; Chen, S.; Li, X.; Qu, C. Ultrasonic-assisted synthesis of chrysin derivatives linked with 1,2,3-triazoles by 1,3-dipolar cycloaddition reaction. Ultrason. Sonochem. 2011, 18, 527-533. [CrossRef] [PubMed]

33. Cintas, P.; Palmisano, G.; Cravotto, G. Power ultrasound in metal-assisted synthesis: From classical Barbier-like reactions to click chemistry. Ultraso. Sonochem. 2011, 18, 836-841. [CrossRef] [PubMed]

34. Mady, M.F.; Awad, G.E.A.; Jørgensen, K.B. Ultrasound-assisted synthesis of novel 1,2,3-triazoles coupled diaryl sulfone moieties by the CuAAC reaction, and biological evaluation of them as antioxidant and antimicrobial agents. Eur. J. Med. Chem. 2014, 84, 433-443. [CrossRef] [PubMed]

35. Akella, S.S.; Kilambi, N.; Lakshmanan, M.; Sriram, R.; Thangapazham, S.; Gaddam, R. Preparation of Acylated Piperazines as Histone Deacetylase (HDAC) Inhibitors for Treating Cancer, Psoriasis and Related Diseases. US Patent 20070088043, 19 April 2007.

36. Zhang, X.; He, Y.; Liu, S.; Yu, Z.; Jiang, Z.-X.; Yang, Z.; Dong, Y.; Nabinger, S.C.; Wu, L.; Gunawan, A.M.; et al. Salicylic Acid Based Small Molecule Inhibitor for the Oncogenic Src Homology-2 Domain Containing Protein Tyrosine Phosphatase-2 (SHP2). J. Med. Chem. 2010, 53, 2482-2493.

37. Committee for Antimicrobial Susceptibility Testing (EUCAST) of the European Society of Clinical Microbiology and Infectious Diseases (ESCMID). Determination of minimum inhibitory concentrations (MICs) of antibacterial agents by agar dilutionEuropean. Clin. Microbiol. Infect. 2000, 6, 509-515.

38. National Committee for Clinical Laboratory Standards. Methods for Dilution Antimicrobial Susceptibility Tests for Bacteria That Grow Aerobically Approved Standard M7-A5, 5th ed.; NCCLS: Wayne, PA, USA, 2000.

Sample Availability: Samples of the compounds are not available from the author.

(C) 2016 by the author; licensee MDPI, Basel, Switzerland. This article is an open access article distributed under the terms and conditions of the Creative Commons Attribution (CC-BY) license (http://creativecommons.org/licenses/by/4.0/). 